\title{
Combining Extremum Seeking Control and Tracking Control for High-Performance CVT Operation
}

\author{
Stan van der Meulen, Bram de Jager, Frans Veldpaus, and Maarten Steinbuch
}

\begin{abstract}
The control design for the variator in a pushbelt continuously variable transmission (CVT) is investigated. The variator enables a stepless variation of the transmission ratio within a finite range. A conventional variator control design is typically obtained by the use of a variator model, which is highly uncertain and, therefore, limits the variator efficiency. In this paper, a variator control design is proposed, which simultaneously satisfies the variator control objectives: 1) tracking a transmission ratio reference, 2) optimizing the variator efficiency. Furthermore, the variator control design, which consists of a combination of extremum seeking control (ESC) and tracking control (TC), only uses measurements from sensors that are standard. Experiments illustrate that the variator control design achieves the variator control objectives and show that a conventional variator control design is outperformed.
\end{abstract}

\section{INTRODUCTION}

The pushbelt continuously variable transmission (CVT) is a stepless power transmission device, which is characterized by the infinite number of transmission ratios within a finite range. Given a power request, this property enables the selection of the transmission ratio for which the fuel consumption of the internal combustion engine (ICE) is minimized. Hence, the fuel consumption is reduced in comparison with a stepped power transmission device [1].

The pushbelt CVT incorporates several components, e.g., the variator and the hydraulic actuation system. The variator consists of a metal V-belt, i.e., a pushbelt, which is clamped between two pairs of conical sheaves, i.e., two pulleys, see Fig. 1. A primary (input, subscript "p") pulley and a secondary (output, subscript "s") pulley are distinguished. Each pulley consists of one axially moveable sheave and one axially fixed sheave. Each axially moveable sheave is connected to a hydraulic cylinder, which is pressurized by the hydraulic actuation system. Essentially, the hydraulic actuation system translates a desired pressure $p_{j \text {,ref }}$ into a realized pressure $p_{j}$, where the pressure $p_{j}$ in the hydraulic cylinder is directly related to the clamping force $F_{j}$ on the axially moveable sheave, where $j \in\{p, s\}$. The level of the clamping forces determines the torque capacity, whereas the ratio of the clamping forces determines the transmission ratio. When the level of the clamping forces is too high, variator efficiency is compromized, since the friction loss is increased. When the level of the clamping forces is too low, torque capacity is compromized. This reduces variator

S. van der Meulen, B. de Jager, F. Veldpaus, and $M$. Steinbuch are with the Department of Mechanical Engineering, Control Systems Technology Group, Eindhoven University of Technology, PO Box 513, 5600 MB Eindhoven, The Netherlands. S.H.v.d.Meulen@tue.nl, A.G.de.Jageratue.nl, F.E.Veldpausetue.nl, M.Steinbuch@tue.nl

This research is partially funded by Bosch Transmission Technology, Tilburg, The Netherlands. efficiency and introduces variator damage. Hence, there exists a choice of the clamping forces that optimizes the variator efficiency, which demands a control design in which the variator efficiency is explicitly addressed.

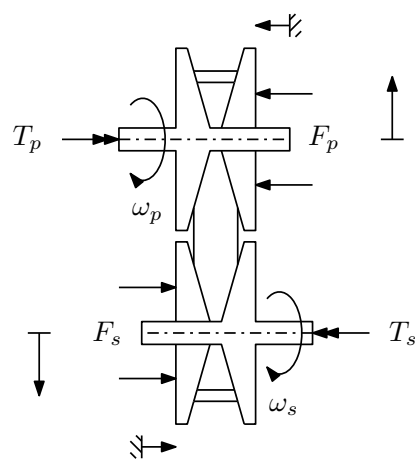

Fig. 1. Schematic illustration of pushbelt variator.

The objective for the variator control system is twofold: 1) tracking a speed ratio reference $r_{s, \text { ref }}$, which is prescribed by the driveline control system; 2) optimizing the variator efficiency $\eta$. The transmission ratio is represented by the speed ratio $r_{s}$, which is easily computed from the ratio of the measurements of the angular velocities. The variator efficiency $\eta$ is defined by the ratio of the powers, which are not measured.

Traditionally, the majority of the approaches control the speed ratio via the primary pulley with the primary hydraulic circuit and the torque capacity via the secondary pulley with the secondary hydraulic circuit, see, e.g., [2]-[4]. The primary pressure that is required in order to achieve the speed ratio is computed by means of a feedback controller (closed loop). Several feedback control designs are encountered, e.g., PI(D) control [2], fuzzy control [3], robust control [4]. The secondary pressure that is required in order to transfer the torque is computed by means of a variator model (open loop). Since the variator model is highly uncertain, a safety strategy is employed, which utilizes a safety factor. Generally, the safety factor ranges from $1.2[-]$ to $1.3[-]$, which implies that the variator efficiency is seriously compromized.

Recently, the variator efficiency is explicitly addressed in the control design that is proposed in [5]. The existence of a certain optimum for the variator efficiency as a function of the slip is shown by means of experiments. As a result, a straightforward approach is to control the slip in such a way that a certain slip reference is tracked, which corresponds to the optimum variator efficiency [5]. However, this approach involves two issues. First, the determination of the slip reference [6, Section 7.2]. Since the optimum variator efficiency 
depends on, e.g., the transmission ratio, the variator load, and the variator wear, the determination of the slip reference is not straightforward and often time-consuming, which is typically caused by the complexity and the unreliability of the available variator models. Second, the reconstruction of the slip in the variator. This typically requires a dedicated sensor, e.g., measurement of the axially moveable sheave position [5], which increases both the complexity and the costs. In addition, the reconstruction of the slip in the variator on the basis of one of these measurements is extremely sensitive to deformations in the variator, which are unknown.

These drawbacks are avoided in the control design that is proposed in [7], [8], which effectively improves the variator efficiency and only uses the measurements of the angular velocities and the secondary pressure, which are standard. The control design exploits the observation that the maximum of the $\left(p_{s}, r_{s}\right)$ equilibrium map and the maximum of the $\left(p_{s}, \eta\right)$ equilibrium map are achieved for secondary pressure values that nearly coincide. This motivates the consideration of the input-output equilibrium map in which the secondary pressure $p_{s}$ is the input and the speed ratio $r_{s}$ is the output, although the location of the maximum is not known. Moreover, the location of the maximum is determined by the operating conditions, which implies that the location of the maximum is not fixed. For these reasons, the maximum of the input-output equilibrium map is found by means of extremum seeking control (ESC) [9], which aims to adapt the input in order to maximize the output. In [7], [8], however, the control problem for optimizing the variator efficiency is isolated from the control problem for tracking the speed ratio reference. With this simplification, a single-input singleoutput (SISO) control problem is obtained (input: $p_{s}$, output: $\left.\partial r_{s} / \partial p_{s}\right)$. Without this simplification, a multi-input multioutput (MIMO) control problem is obtained (inputs: $p_{p}$ and $p_{s}$, outputs: $r_{s}$ and $\left.\partial r_{s} / \partial p_{s}\right)$, which is not treated, yet.

The main contribution of this paper concerns a solution for the MIMO control problem that simultaneously satisfies both variator control objectives, which is successfully demonstrated by means of experiments. This is achieved via the integration of the ESC design and a tracking control (TC) design. The remainder of this paper is organized as follows. The preliminaries are addressed in Section II, which includes several definitions, the experimental setup, and the control problem formulation. The ESC design is described in Section III. The TC design is introduced in Section IV. In Section V, a closed loop experiment shows that the variator control objectives are satisfied. Finally, the paper concludes with a discussion in Section VI.

Notation: Consider the variator that is depicted in Fig. 1. The torques that are exerted on the variator are denoted by $T_{p}$ and $T_{s}$. Furthermore, $\omega_{p}$ and $\omega_{s}$ are the angular velocities and $F_{p}$ and $F_{s}$ are the clamping forces.

\section{PRELIMinaries}

\section{A. Definitions}

The speed ratio $r_{s}$ of the variator is defined by:

$$
r_{s}=\frac{\omega_{s}}{\omega_{p}}
$$

The variator efficiency $\eta$ is defined by:

$$
\eta=\frac{P_{\text {out }}}{P_{\text {in }}}=\frac{T_{s} \omega_{s}}{T_{p} \omega_{p}},
$$

where $P_{\text {in }}$ and $P_{\text {out }}$ denote the input power and the output power, respectively.

\section{B. Experimental Setup}

The experimental setup is depicted in Fig. 2 and consists of five main components. These are given by two identical electric motors, a pushbelt variator, a hydraulic actuation system, and a data acquisition system. The experimental setup incorporates additional sensors in comparison with a production CVT, which are only used for analysis purposes.

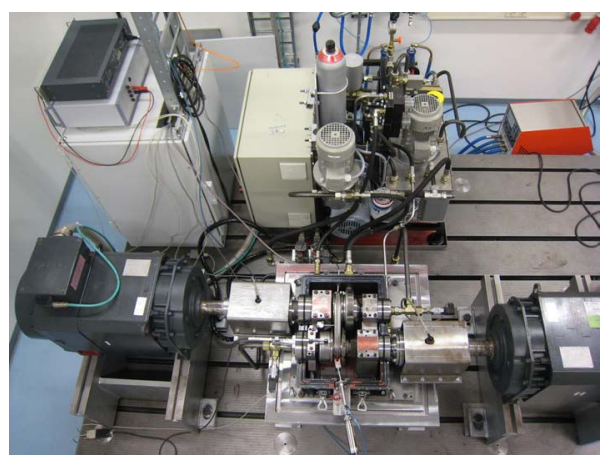

Fig. 2. Experimental setup with pushbelt variator.

\section{Control Problem Formulation}

Consider the control configuration that is depicted in Fig. 3. A cascade control design is employed. The inner loop includes the hydraulic actuation system, which is closed loop controlled [10]. The desired pressures $p_{p \text {, ref }}$ and $p_{s, \text { ref }}$ are the inputs and the realized pressures $p_{p}$ and $p_{s}$ are the outputs, which are related by:

$$
\left[\begin{array}{c}
p_{p} \\
p_{s}
\end{array}\right]=\left[\begin{array}{ll}
T_{H_{p p}} & T_{H_{p s}} \\
T_{H_{s p}} & T_{H_{s s}}
\end{array}\right]\left[\begin{array}{l}
p_{p, \text { ref }} \\
p_{s, \text { ref }}
\end{array}\right] .
$$

The outer loop includes the variator, which is controlled by the combination of ESC and TC. The interconnection between the pressures $p_{p}$ and $p_{s}$ and the speed ratio $r_{s}$ is defined by:

$$
\left[r_{s}\right]=\left[\begin{array}{ll}
G_{V_{p}} & G_{V_{s}}
\end{array}\right]\left[\begin{array}{l}
p_{p} \\
p_{s}
\end{array}\right] .
$$

The control problem formulations for the ESC design and the TC design are subsequently defined.

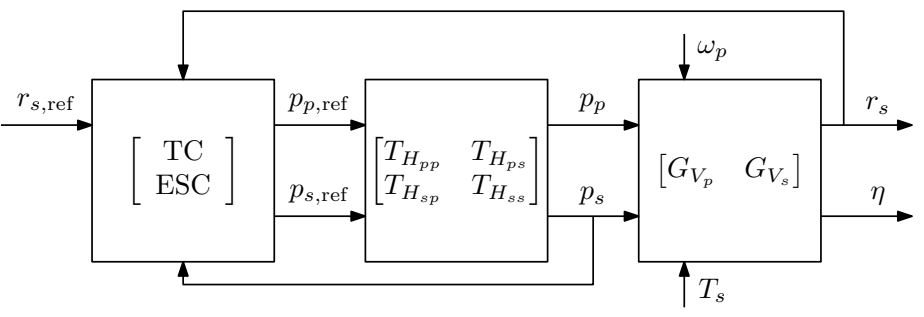

Fig. 3. Control configuration. 
1) ESC Problem Formulation: Consider the SISO nonlinear system:

$$
\begin{aligned}
u & =p_{s} \\
u_{\mathrm{ref}} & =p_{s, \mathrm{ref}} \\
y & =r_{s} \\
\dot{y} & =f_{s}\left(y, u_{\mathrm{ref}}, \omega_{p}, T_{s}\right),
\end{aligned}
$$

where $f_{s}$ is smooth. The following assumptions are introduced with respect to the SISO nonlinear system (5).

Assumption 1 There exists a smooth function $h: \mathbb{R} \rightarrow \mathbb{R}$ such that:

$$
\dot{y}=0 \Leftrightarrow y=h\left(u_{\mathrm{ref}}\right) .
$$

The equilibrium $y=h\left(u_{\mathrm{ref}}\right)$ is exponentially stable.

Assumption 2 There exists $u_{\text {ref }}^{*} \in \mathbb{R}$ such that:

$$
\begin{aligned}
& \left.\frac{\partial h}{\partial u_{\text {ref }}}\right|_{u_{\text {ref }=u_{\text {ref }}^{*}}}=0 \\
& \left.\frac{\partial^{2} h}{\partial u_{\text {ref }}^{2}}\right|_{u_{\text {ref }}=u_{\text {ref }}^{*}}<0 .
\end{aligned}
$$

That is, the input-output equilibrium map $y=h\left(u_{\mathrm{ref}}\right)$ has a maximum for $u_{\mathrm{ref}}=u_{\mathrm{ref}}^{*}$.

These assumptions are satisfied for the operating conditions that normally occur, see [8]. The following objective is formulated with respect to the SISO nonlinear system (5).

Objective 3 Design a feedback mechanism from y to $u_{\text {ref }}$ without the knowledge of both $u_{\mathrm{ref}}^{*}$ and the function $h$ that ensures that the steady-state value of $y$ is maximized.

2) TC Problem Formulation: Consider the SISO nonlinear system:

$$
\begin{aligned}
u & =p_{p} \\
u_{\mathrm{ref}} & =p_{p, \mathrm{ref}} \\
y & =r_{s} \\
\dot{y} & =f_{p}\left(y, u_{\mathrm{ref}}, \omega_{p}, T_{s}\right),
\end{aligned}
$$

where $f_{p}$ is smooth. The following objective is formulated with respect to the SISO nonlinear system (9).

Objective 4 Design a feedback mechanism from y to $u_{\mathrm{ref}}$ that ensures that the speed ratio reference $r_{s, \mathrm{ref}}$ is asymptotically tracked by $y$.

\section{Extremum Seeking Control Design}

\section{A. ESC Design}

The feedback mechanism is depicted in Fig. 4. Obviously, the feedback mechanism utilizes a sinusoidal perturbation $\alpha_{m} \sin \left(2 \pi f_{m} t\right)$, which is added to $\hat{u}_{\text {ref }}$, i.e., the estimate of the optimum input $u_{\text {ref }}^{*}$. As a result, the input of the hydraulic actuation system $u_{\text {ref }}$ is defined by:

$$
u_{\text {ref }}(t)=\hat{u}_{\text {ref }}(t)+\alpha_{m} \sin \left(2 \pi f_{m} t\right),
$$

where $\alpha_{m}$ denotes the perturbation amplitude and $f_{m}$ denotes the perturbation frequency. When $\hat{u}_{\text {ref }}$ is on either side of $u_{\mathrm{ref}}^{*}$, the periodic perturbation enforces a periodic response of the output of the variator $y$, which is either in phase or out of phase with the periodic perturbation. With this information, the feedback mechanism from $y$ to $\hat{u}_{\text {ref }}$ is designed, which consists of the following operations:

$$
\begin{aligned}
\xi_{1} & =H_{b}(s) y \\
\xi_{2} & =H_{b}(s) u \\
\xi_{3} & =\xi_{1} \xi_{2} \\
\xi_{4} & =H_{l}(s) \xi_{3} \\
\hat{u}_{\text {ref }} & =\frac{1}{s} I \xi_{4} .
\end{aligned}
$$

Here, $H_{b}(s)$ denotes a band-pass filter, $H_{l}(s)$ denotes a lowpass filter, and $I$ denotes the integrator gain. The band-pass filter $H_{b}(s)$ enforces the suppression of "DC components" and noise for $y$ and $u$, which results in $\xi_{1}$ and $\xi_{2}$, respectively. As a result, $\xi_{1}$ and $\xi_{2}$ are approximately two sinusoids, which are out of phase for $\hat{u}_{\text {ref }}>u_{\text {ref }}^{*}$ and in phase for $\hat{u}_{\text {ref }}<u_{\text {ref }}^{*}$. In either case, the product of both sinusoids $\xi_{3}$ has a "DC component". The low-pass filter $H_{l}(s)$ extracts the "DC component" of $\xi_{3}$, which results in $\xi_{4}$. Finally, $\hat{u}_{\text {ref }}$ results from integration of $\xi_{4}$, with integrator gain $I$. The initial condition for the integrator is equal to $\bar{u}_{\text {ref }}$, which corresponds to a stationary operating point. Observe that (14) contains the gradient information and (15) represents the gradient update law, which enables the adaptation of $\hat{u}_{\text {ref }}$ towards the optimum input $u_{\mathrm{ref}}^{*}$.

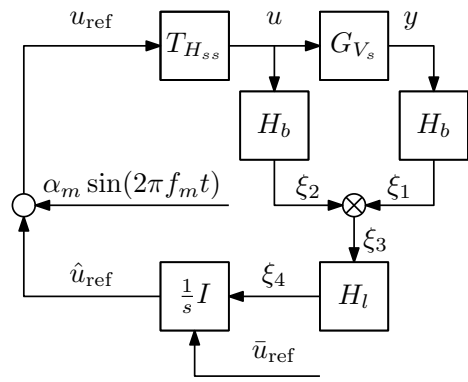

Fig. 4. Feedback mechanism from $y$ to $u_{\text {ref }}$ for ESC.

Obviously, the feedback mechanism incorporates five design options. These are the perturbation amplitude $\alpha_{m}$, the perturbation frequency $f_{m}$, the band-pass filter $H_{b}(s)$, the low-pass filter $H_{l}(s)$, and the integrator gain $I$. The selection of these design options is closely related to the proof of stability for the closed loop system, which is addressed in [9]. The feedback mechanism in [9] is similar to the feedback mechanism in Fig. 4. However, a high-pass filter is employed instead of a band-pass filter. The main reason for the application of a band-pass filter concerns the suppression of noise. When Assumptions 1 and 2 are satisfied, convergence of the solution $\left(\hat{u}_{\text {ref }}(t), \xi_{4}(t), y(t)\right)$ towards a certain neighborhood of the point $\left(u_{\mathrm{ref}}^{*}, 0, h\left(u_{\mathrm{ref}}^{*}\right)\right)$ is guaranteed by [9, Theorem 5.1] for a suitable choice of the design options. A suitable choice of the design options is made in Section III-B.

\section{B. Design Options}

The perturbation amplitude $\alpha_{m}$, the perturbation frequency $f_{m}$, the band-pass filter $H_{b}(s)$, the low-pass filter $H_{l}(s)$, and 
the integrator gain $I$ are given by:

$$
\begin{aligned}
\alpha_{m} & =0.7 \\
f_{m} & =10 \\
H_{b}(s) & =\frac{\frac{2}{2 \pi f_{m}} 0.003 s}{\frac{1}{\left(2 \pi f_{m}\right)^{2}} s^{2}+\frac{2}{2 \pi f_{m}} 0.003 s+1} \\
H_{l}(s) & =\frac{1}{\frac{1}{2 \pi 1} s+1} \\
I & =1250 .
\end{aligned}
$$

The Bode magnitude diagrams of the band-pass filter $H_{b}(s)$ and the low-pass filter $H_{l}(s)$ are depicted in Fig. 5. Both filters are discretized on the basis of a first-order hold discretization scheme.

Upper bounds are imposed on the perturbation amplitude $\alpha_{m}$ and the perturbation frequency $f_{m}$, in order to confine the size of the region to which the solution converges [9, Theorem 5.1]. On the other hand, a sufficiently large $\alpha_{m}$ and $f_{m}$ are required in order to excite the variator and to achieve convergence, respectively, see [11, Section 1.2.3]. The band-pass filter $H_{b}(s)$ is designed in accordance with the perturbation frequency $f_{m}$ and enforces the suppression of "DC components" and noise. The cut-off frequency of the low-pass filter $H_{l}(s)$ is a fraction of the perturbation frequency $f_{m}$, see [9]. Finally, the integrator gain $I$ is limited in order to confine the size of the region to which the solution converges [9, Theorem 5.1]. On the other hand, a sufficiently large $I$ is desired in order to accelerate convergence.
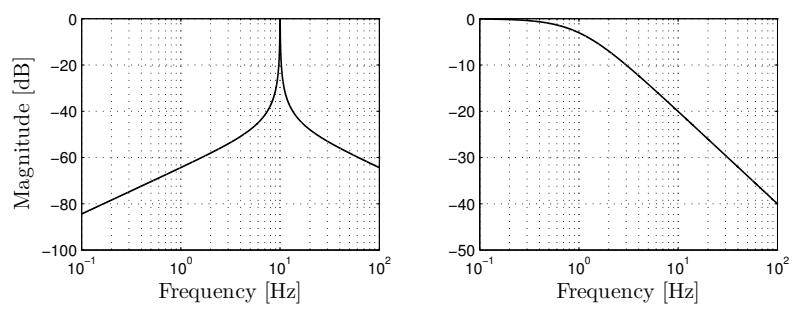

Fig. 5. Bode magnitude diagrams of filters (Left: Band-pass filter $H_{b}(s)$; Right: Low-pass filter $H_{l}(s)$ ).

\section{Tracking Control Design}

Since the system dynamics of the hydraulic actuation system and the variator are fairly complex, modeling via first principles is hampered. Therefore, modeling via system identification is pursued, which provides a nonparametric model, see Section IV-A. On the basis of this nonparametric model, the TC design is constructed via loop-shaping, see Section IV-B.

\section{A. Nonparametric System Identification}

Consider the configuration for the system identification problem that is depicted in Fig. 6. Here, $\Delta p_{p \text {,ref }}$ and $\Delta p_{s, \text { ref }}$ denote the deviations that are possibly superposed to the nominal values $\bar{p}_{p \text {,ref }}$ and $\bar{p}_{s \text {,ref. }}$. Furthermore, $d$ denotes the disturbances, e.g., noise. The system identification problem is to find a representation for the nonlinear system between the input $u$ and the output $y$, which are defined by:

$$
\begin{aligned}
u & =\Delta p_{p, \text { ref }} \\
y & =r_{s}-\bar{r}_{s},
\end{aligned}
$$

where $\bar{r}_{s}$ denotes the mean of $r_{s}$. Obviously, this system identification problem is of the open loop type, see Fig. 6, which enables the application of well-established techniques from the system identification field [12].

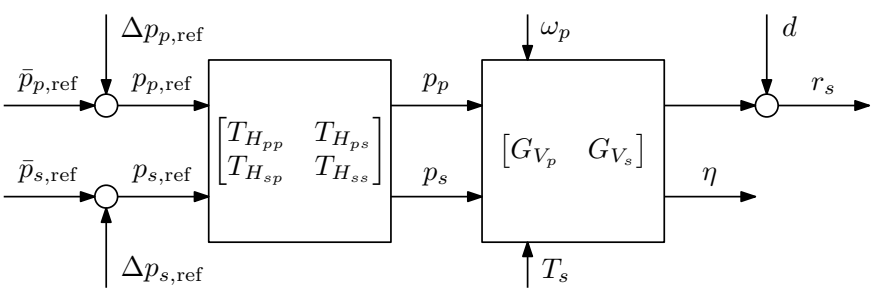

Fig. 6. Configuration for system identification problem.

When the operating conditions of the hydraulic actuation system and the variator are varied, the system dynamics are significantly changed. When these variations of the system dynamics are neglected in the control design, this probably deteriorates the control performance or possibly destabilizes the closed loop. Here, the variations of the system dynamics are addressed via a set of local linear models, which is obtained from a sequence of identification experiments. The sequence of identification experiments encompasses $20 \mathrm{com}$ binations of the operating conditions, which are determined by $\bar{p}_{p, \text { ref }}, \bar{p}_{s, \text { ref }}, \omega_{p}$ or $\omega_{s}$, and $T_{p}$ or $T_{s}$. Here, 6 combinations of the operating conditions are selected, see Table I, from which the set of local linear models is constructed. This set of local linear models is representative for the system dynamics within the range that is normally covered.

TABLE I

OPERATING CONDITIONS FOR IDENTIFICATION EXPERIMENTS.

\begin{tabular}{l|l|l|l|l}
\hline Number & $\bar{p}_{p, \text { ref }}[\mathrm{bar}]$ & $\bar{p}_{s, \text { ref }}[\mathrm{bar}]$ & $\omega_{s}[\mathrm{rpm}]$ & $T_{p}[\mathrm{Nm}]$ \\
\hline 1 & 6.0 & 30.0 & 2900 & -44 \\
2 & 5.0 & 17.0 & 2900 & -39 \\
3 & 6.0 & 15.5 & 2900 & -37 \\
4 & 6.0 & 15.5 & 3500 & -39 \\
5 & 6.0 & 12.7 & 2900 & -38 \\
6 & 6.0 & 12.7 & 3500 & -37 \\
\hline
\end{tabular}

The excitation signal $u$ is either periodic or nonperiodic. The use of periodic excitation signals is strongly advocated in [12], in view of frequency response function (FRF) measurements that are low-cost and high-quality. The main advantage of periodic excitation signals in comparison with nonperiodic excitation signals is given by the possibility of reducing variance by means of averaging techniques, without introducing bias. Here, the multisine is applied, i.e., a sum of sine waves that are harmonically related, which is a periodic excitation signal. The multisine is defined by:

$$
u(t)=\sum_{k=1}^{F} \alpha_{k} \cos \left(2 \pi f_{k} t+\varphi_{k}\right),
$$


where the index $k$ denotes the sine wave. Furthermore, $F$ denotes the number of frequencies, $f_{k}$ the frequency, $\alpha_{k}$ the amplitude, and $\varphi_{k}$ the phase. The Schroeder phases are used [12, Section 4.3.1.2], which are defined by:

$$
\varphi_{k}=\frac{-k(k-1) \pi}{F}
$$

for which the crest factor, i.e., the ratio between the peak value of $u(t)$ and the root mean square (RMS) value of $u(t)$, is fairly low.

The sampling frequency is equal to $f_{s}=1000[\mathrm{~Hz}]$ and the frequency resolution is equal to $f_{1}=0.05[\mathrm{~Hz}]$. The number of frequencies $F$ in the discrete frequency grid $\Omega\left(f_{k}\right)$ is equal to $F=41[-]$, where $\Omega\left(f_{k}\right), k=1, \ldots, F$, is given by $\Omega\left(f_{k}\right) \in\{0.05, \ldots, 48\}[\mathrm{Hz}]$. Moreover, the lowpass character of the system requires that the amplitude $\alpha_{k}$ is nonuniformly chosen, in order to ensure that the system is adequately excited. Although the number of frequencies $F$ is limited, the damped character of the system suggests that the discrete frequency grid $\Omega\left(f_{k}\right)$ is sufficiently dense. Finally, for each of the operating conditions, the number of fundamental periods $M$ is equal to $M=44$ [-]. The measurements of the input $u$ and the output $y$ for each of the fundamental periods are transformed to the frequency domain via the discrete Fourier transform (DFT), averaged, and divided. This provides the FRF, which is a nonparametric model.

The set of FRFs $P\left(\jmath \omega_{k}\right)$, which consists of $P_{i}\left(\jmath \omega_{k}\right), i=$ $1, \ldots, 6$, is depicted in Fig. 7. This set of FRFs $P\left(\jmath \omega_{k}\right)$ is representative for the system dynamics within the range that is normally covered. The confidence intervals are omitted, since the variance errors are negligible. The variations of the system dynamics are clearly visible. Furthermore, the lowpass character of the system and the damped character of the system are indisputably confirmed.
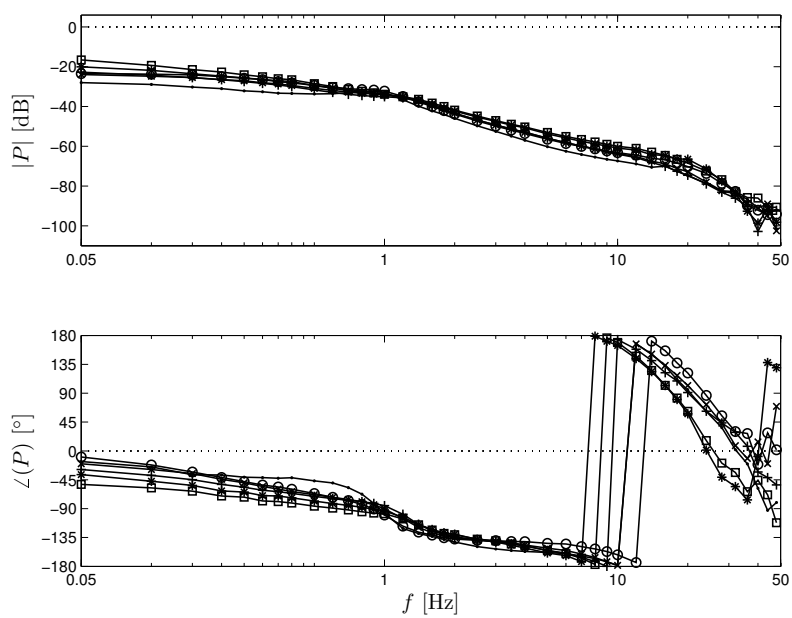

Fig. 7. Set of FRFs $P\left(\jmath \omega_{k}\right)(\because: 1 ; \quad \circ: 2 ; \quad \times: 3 ; \quad+: 4 ; \quad *: 5 ; \quad \square: 6)$.

\section{B. TC Design}

On the basis of the set of FRFs $P\left(\jmath \omega_{k}\right)$ that is depicted in Fig. 7, the TC design $K(s)$ is constructed via loopshaping [13]. The TC design $K(s)$ is given by the product of the following parts:

$$
\begin{aligned}
K_{\text {gain }} & =3 \cdot 10^{5} \\
K_{\text {int }}(s) & =\frac{1}{s} \\
K_{\text {lead }}(s) & =\frac{\left(\frac{1.1}{2 \pi 1} s+1\right)^{2}}{1} \\
K_{\text {notch }}(s) & =\frac{\frac{1}{\left(2 \pi f_{m}\right)^{2}} s^{2}+\frac{2}{2 \pi f_{m}} 0.002 s+1}{\frac{1}{\left(2 \pi f_{m}\right)^{2}} s^{2}+\frac{2}{2 \pi f_{m}} 0.05 s+1} \\
K_{\text {roll-off }}(s) & =\frac{1}{(s+2 \pi 6)^{2}},
\end{aligned}
$$

which stabilizes the set of FRFs $P\left(\jmath \omega_{k}\right)$. The notch filter $(24 \mathrm{~d})$ is implemented in order to reduce the suppression of the periodic response that is enforced by the sinusoidal perturbation of the ESC design. The Bode magnitude diagram of the TC design $K(s)$ is depicted in Fig. 8. The TC design $K(s)$ is discretized on the basis of a first-order hold discretization scheme.

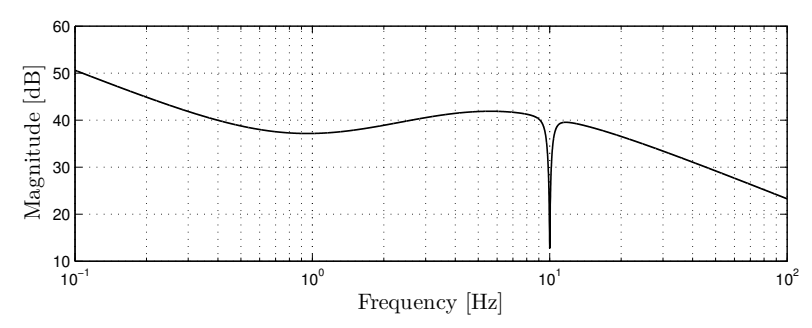

Fig. 8. Bode magnitude diagram of TC design $K(s)$.

\section{Closed LoOp EXPERIMENT}

The operation of the combination of the ESC design and the TC design is evaluated by means of a closed loop experiment, see Fig. 3. The operating conditions are given by $\omega_{p}=1000[\mathrm{rpm}]$ and $T_{s}=20[\mathrm{Nm}]$, whereas the speed ratio reference is equal to $r_{s, \text { ref }}=1.2$ [-]. The closed loop experiment is started from a stationary operating point, which is defined by the initial condition for the integrator, see Fig. 4. The initial condition for the integrator is equal to $\bar{p}_{s, \text { ref }}=7.2$ [bar], which corresponds to the secondary pressure reference $p_{s, \text { ref }}$ that is achieved by the absolute safety strategy, where the absolute safety factor is equal to 1.3 [-]. This absolute safety strategy is commonly used by the automotive industry, see [14]. For this reason, this absolute safety strategy is adopted for comparison purposes.

The experimental results for the proposed strategy and the absolute safety strategy are depicted in Figs. 9, 10, and 11. From Fig. 9 (bottom left), it follows that the ESC feedback mechanism converges from $\bar{p}_{s \text {,ref }}$ to $p_{s, \text { ref }}^{*}$, which is approximately reached for $t \approx 250$ [s]. The convergence speed is reasonably low, which is possibly improved by extensions of the ESC feedback mechanism, see, e.g., [15]. Furthermore, the sinusoidal perturbation is clearly visible, which is sufficiently small in order to avoid problems with noise, vibration, and harshness (NVH). From Fig. 9 (top left), it follows that the TC feedback mechanism adjusts the primary pressure reference $p_{p \text {,ref }}$ in order to track the speed 

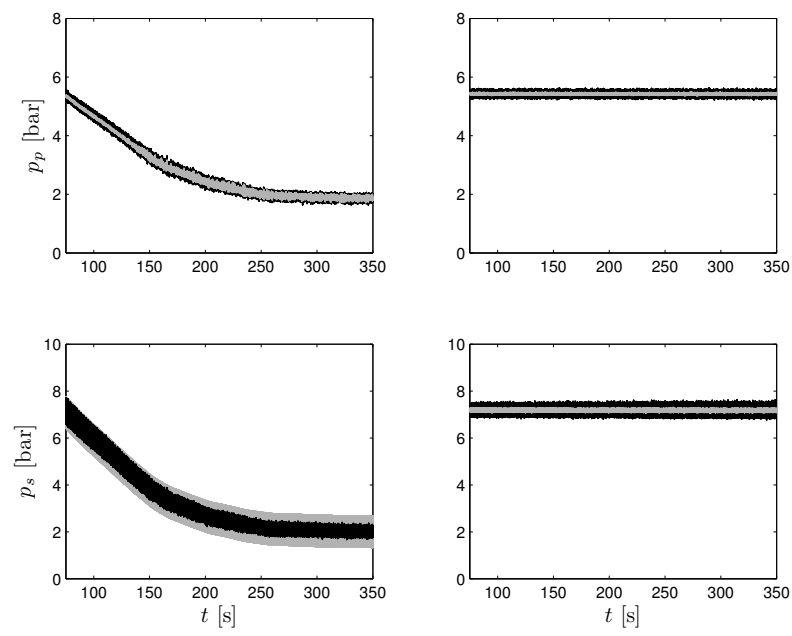

Fig. 9. Experimental results for pressures (Left: Proposed strategy; Right: Absolute safety strategy) (black: Measurement; grey: Reference).
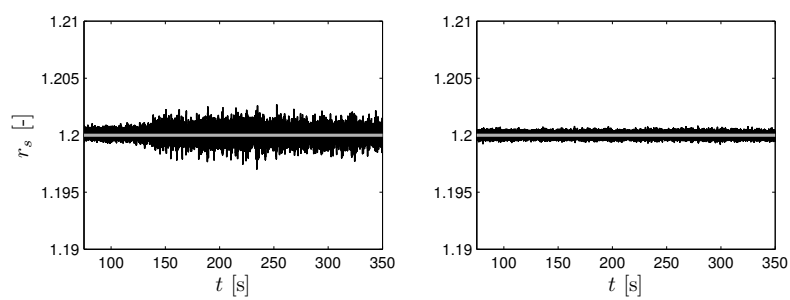

Fig. 10. Experimental results for speed ratio (Left: Proposed strategy; Right: Absolute safety strategy) (black: Measurement; grey: Reference).

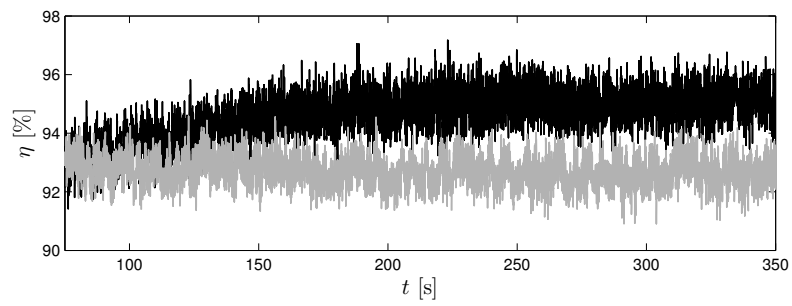

Fig. 11. Experimental results for variator efficiency (black: Proposed strategy; grey: Absolute safety strategy).

ratio reference $r_{s, \text { ref }}$. Obviously, the primary pressure reference $p_{p \text {,ref }}$ decreases, since the secondary pressure reference $p_{s, \text { ref }}$ decreases. From Fig. 9 (right), it follows that both the primary pressure reference $p_{p, \text { ref }}$ and the secondary pressure reference $p_{s, \text { ref }}$ are stationary for the absolute safety strategy. From Fig. 10, it follows that the speed ratio reference $r_{s, \text { ref }}$ is accurately tracked for both strategies. Finally, the variator efficiency $\eta$ for both the proposed strategy and the absolute safety strategy is depicted in Fig. 11. Obviously, when the pressure references for the proposed strategy decrease in comparison with the absolute safety strategy, the variator efficiency increases. The gain with respect to the variator efficiency is approximately equal to 2.5 [\%].

\section{DISCUSSION}

In this paper, a control design for the variator in a pushbelt CVT is proposed that effectively improves the variator efficiency and only uses the measurements of the angular velocities and the secondary pressure, which are standard. The variator control design, which consists of a combination of extremum seeking control (ESC) and tracking control (TC), simultaneously satisfies both objectives: 1) tracking a speed ratio reference; 2) optimizing the variator efficiency. This is successfully demonstrated by means of experiments, where the speed ratio reference is fixed. The experiments show that the absolute safety strategy, which is commonly used by the automotive industry, is outperformed.

Several opportunities for future research are recognized. The performance of the ESC design is improved when the convergence speed is increased, via optimization or extension of the ESC design. The performance of the TC design is improved when the variations of the system dynamics are explicitly considered, via feedback linearization or gain scheduling, for example. Finally, further research is required with respect to tracking a speed ratio reference that is obtained from a driving cycle and suppressing a torque disturbance that is induced by the ICE or the road.

\section{REFERENCES}

[1] R. Pfiffner and L. Guzzella, "Optimal operation of CVT-based powertrains," Int. J. Rob. Nonlin. Contr., vol. 11, no. 11, pp. 1003-1021, 2001.

[2] S. Hirano, A. L. Miller, and K. F. Schneider, "SCVT - A State of the Art Electronically Controlled Continuously Variable Transmission," SAE Tech. Pap. Ser., no. 910410, 1991.

[3] W. Kim and G. Vachtsevanos, "Fuzzy Logic Ratio Control for a CVT Hydraulic Module," in Proc. 2000 IEEE Int. Symp. Intell. Contr. vol. 1, Rio, Patras, Greece, 2000, pp. 151-156.

[4] K. Adachi, T. Wakahara, S. Shimanaka, M. Yamamoto, and T. Oshidari, "Robust control system for continuously variable belt transmission," JSAE Rev., vol. 20, no. 1, pp. 49-54, 1999.

[5] B. Bonsen, T. W. G. L. Klaassen, R. J. Pulles, S. W. H. Simons, M. Steinbuch, and P. A. Veenhuizen, "Performance optimisation of the push-belt CVT by variator slip control," Int. J. Veh. Des., vol. 39, no. 3, pp. 232-256, 2005.

[6] T. W. G. L. Klaassen, "The Empact CVT: Dynamics and Control of an Electromechanically Actuated CVT," Ph.D. Thesis, Eindhoven University of Technology, Eindhoven, The Netherlands, 2007.

[7] E. van der Noll, F. van der Sluis, T. van Dongen, and A. van der Velde, "Innovative Self-optimising Clamping Force Strategy for the Pushbelt CVT," in Proc. SAE 2009 World Congr., no. 2009-01-1537, Detroit, MI, 2009, CD-ROM.

[8] S. van der Meulen, B. de Jager, E. van der Noll, F. Veldpaus, F. van der Sluis, and M. Steinbuch, "Improving Pushbelt Continuously Variable Transmission Efficiency via Extremum Seeking Control," in Proc. 3rd IEEE Multi-conf. Syst. Contr., Saint Petersburg, Russia, 2009, pp. 357362.

[9] M. Krstić and H.-H. Wang, "Stability of extremum seeking feedback for general nonlinear dynamic systems," Automatica, vol. 36, no. 4, pp. 595-601, 2000.

[10] T. Oomen, S. van der Meulen, O. Bosgra, M. Steinbuch, and J. Elfring, "A Robust-Control-Relevant Model Validation Approach for Continuously Variable Transmission Control," in Proc. 2010 Amer. Contr. Conf., Baltimore, MD, 2010, pp. 3518-3523.

[11] K. B. Ariyur and M. Krstić, Real-Time Optimization by ExtremumSeeking Control. Hoboken, New Jersey: John Wiley \& Sons, 2003.

[12] R. Pintelon and J. Schoukens, System Identification: A Frequency Domain Approach. New York: The Institute of Electrical and Electronics Engineers, 2001.

[13] S. Skogestad and I. Postlethwaite, Multivariable Feedback Control: Analysis and Design, 2nd ed. Chichester, West Sussex, England: John Wiley \& Sons, 2005.

[14] F. van der Sluis, T. van Dongen, G.-J. van Spijk, A. van der Velde, and A. van Heeswijk, "Fuel Consumption Potential of the Pushbelt CVT," in Proc. FISITA 2006 World Automotive Congr., no. F2006P218, Yokohama, Japan, 2006, CD-ROM.

[15] M. Krstić, "Performance improvement and limitations in extremum seeking control," Syst. Contr. Lett., vol. 39, no. 5, pp. 313-326, 2000. 